\title{
DESIGN AND SYNTHESIS OF NEW HETEROCYCLIC COMPOUNDS TO OVERCOME MICROBIAL RESISTANCE
}

\author{
Ahmed M. Helal, Abdelrahman S. Mayhoub, Hamada S. Abu-Elkhair*, Ashraf H. \\ Bayoumi. \\ Department of Pharmaceutical Organic Chemistry, Faculty of Pharmacy, Al-Azhar University, \\ Cairo, Egypt
}

*Corresponding author: Ahme_helal@azhar.edu.eg

\begin{abstract}
Though clinicians once possessed a robust arsenal of antibiotics, many of these valuable therapeutic agents have fallen prey to the expanded resistance of pathogenic bacteria. Phenylthiazoles were reported previously as a new scaffold that possesses antibacterial activity against an array of clinically-relevant strains of multidrug-resistant staphylococci. Close inspection of the structure-activity-relationships (SAR) of phenylthiazoles revealed important structural features necessary for their antibacterial activity: a nitrogenous head and a lipophilic tail. Incorporating the nitrogenous part within an oxadiazole ring resulted in analogues with a prolonged half-life, while the biphenyl tail revealed the most potent analogue. In the present work, advantageous moieties have been combined together to generate new hybrid scaffolds of phenylpyridine with the objective of promoting new moieties enhancing both antimicrobial resistance activity and drug-like properties. Among the tested oxadiazolylbiphenylpyridines, derivatives 14 and 23 were identified as the most potent analogues with MIC values as low as $8 \mu \mathrm{g} / \mathrm{mL}$ on MRSA-US300 and other studied species.
\end{abstract}

Keywords: Biphenylpyridine, Antimicrobial resistance, Antibiotics, Multidrugresistant, Synthesis

\section{INTRODUCTION}

The search for new antibiotics has improved human health status by suppressing life-threatening infections. However, the emergence and spread of bacterial resistance represent a severe global problem; the number of life-threatening infectious diseases caused by multidrug-resistant bacteria has reached an alarming level in many countries around the world. (Parmar et al., 2003) Hence, there is both substantial need and market for more effective treatments of bacterial and fungal infections. Novel solutions to the issue are however necessary since the currently used antimicrobial agents are no longer sufficient to prevent certain infections from propagating and spreading due to resistance development. One way to overcome the rapid development of drug resistance to the currently used antimicrobials is to develop new agents, preferably with chemical characteristics that vastly differ from those of existing ones.(Desai et al., 2014) Phenylthiazoles were reported as a new scaffold acting as dual UppP and UppS inhibitors with wide antimicrobial activity against multidrug-resistant gram-positive strains including MRSA, VRSA and VRE(Haroon Mohammad et al., 2014a) with 
selective advantage over vancomycin.(H. Mohammad, Mayhoub, Cushman, \& Seleem, 2015; Haroon Mohammad et al., 2014b) The SAR of this class of compounds is welldefined through more than 400 phenylthiazoles published derivatives. (Pankey \& Sabath, 2004)'(French, 2006)

Pyridine plays a key role in catalyzing both biological and chemical systems. In many enzymes of living organisms it is the prosthetic pyridine nucleotide (NADP) that is involved in various oxidation-reduction processes. Pyridines showed potent antimicrobial activity against wide range of microorganisms.(Prakash et al., 2011) Finally, we wish to report here the synthesis of a new series of oxadiazolylbiphenylpyridine scaffold possessing anti-microbial activities.

\section{Chemistry}

All melting points were carried on Gallen Kamp point apparatus and are uncorrected. ${ }^{1} \mathrm{HNMR}$ spectra were recorded or Bruker-400-MHz spectrophotometer using DMSO- $\mathrm{d}_{6}$ as a solvent and TMS as internal reference. Chemical shift values were recorded in $\delta$ ppm downfield the TMS signal. Mass spectra were recorded on AZH-ph$\mathrm{AR}-\mathrm{XO}_{2}$ Mass spectrometer. Elemental analyses were performed on $\mathrm{CHN}$ analyzer. All spectral measurements have been performed at the Micro analytical Center, Zagazig University, Egypt.

\section{II-1. Experimental}

\section{$\underline{\text { 1-([1,1'-biphenyl]-4-yl)-3-(dimethylamino)prop-2-en-1-one (1) }}$}

To 4-phenyl acetophenone ( $3 \mathrm{~g}, 15.2 \mathrm{mmol}$ ), DMF-DMA (9.4 mL, $4.8 \mathrm{~g}, 30.4$ mmol) was added and the reaction mixture was heated at $80^{\circ} \mathrm{C}$ for $8 \mathrm{~h}$. After cooling, the formed solid was collected by filtration, washed with petroleum ether and crystallized from ethanol to yield the desired product as a yellow solid $(3.6 \mathrm{~g}, 95 \%) \mathrm{mp}$ $=159{ }^{\circ} \mathrm{C} .{ }^{1} \mathrm{H}$ NMR $\left(400 \mathrm{MHz}, \mathrm{DMSO}_{-}\right) \delta 7.99(\mathrm{~d}, J=8.3 \mathrm{~Hz}, 2 \mathrm{H}), 7.75-7.72(\mathrm{~m}$, $5 \mathrm{H}), 7.51(\mathrm{t}, J=7.7 \mathrm{~Hz}, 2 \mathrm{H}), 7.47(\mathrm{t}, J=7.5 \mathrm{~Hz}, 1 \mathrm{H}), 5.88(\mathrm{~d}, J=12.4 \mathrm{~Hz}, 1 \mathrm{H}), 3.1$ $(\mathrm{s}, 3 \mathrm{H}), 2.9(\mathrm{~s}, 3 \mathrm{H}) ;{ }^{13} \mathrm{C}$ NMR (101 MHz, DMSO-d $\left.\mathrm{d}_{6}\right) \delta 185.6,154.6,142.7,139.9$, $139.5,129.4,128.3,127.5,127.2,126.8,91.4,55.3 ; \mathrm{MS}(\mathrm{m} / \mathrm{z}) 251$.

\section{Ethyl-6-([1,1'-biphenyl]-4-yl)-2-methylnicotinate. (2)}

To a solution of the enaminone 1 ( $3 \mathrm{~g}, 12 \mathrm{mmol})$ in glacial acetic acid $(35 \mathrm{~mL})$, ethyl acetoacetate $(10.9 \mathrm{~g}, 10.7 \mathrm{ml}, 84 \mathrm{mmol})$ and ammonium acetate $(9.24 \mathrm{~g}, 120$ mmol) were added. The reaction mixture was heated under reflux overnight. After cooling and pouring into ice-water, the residue obtained was filtered and washed with petroleum ether then with water and finally crystallized from ethanol. Light green solid (3.42 g, 90\%) $\mathrm{mp}=110{ }^{\circ} \mathrm{C} ;{ }^{1} \mathrm{H}$ NMR $\left(400 \mathrm{MHz}, \mathrm{DMSO}_{6}\right) \delta 8.27(\mathrm{~d}, J=8.4 \mathrm{~Hz}$, 2H), $8.24(\mathrm{~d}, J=7.7 \mathrm{~Hz}, 1 \mathrm{H}), 7.99(\mathrm{~d}, J=8.3 \mathrm{~Hz}, 1 \mathrm{H}), 7.83(\mathrm{~d}, \mathrm{~J}=8.3 \mathrm{~Hz}, 2 \mathrm{H}), 7.75$ $(\mathrm{d}, J=7.2 \mathrm{~Hz}, 2 \mathrm{H}), 7.52(\mathrm{t}, J=7.2 \mathrm{~Hz}, 2 \mathrm{H}), 7.42(\mathrm{t}, J=7.2 \mathrm{~Hz}, 1 \mathrm{H}), 4.36(\mathrm{q}, J=7.2$ $\mathrm{Hz}, 2 \mathrm{H}), 2.81(\mathrm{~s}, 3 \mathrm{H}), 1.36(\mathrm{t}, J=7.2 \mathrm{~Hz}, 3 \mathrm{H}) ;{ }^{13} \mathrm{C}$ NMR $\left(101 \mathrm{MHz}, \mathrm{DMSO}-\mathrm{d}_{6}\right)$ $\delta 165.90,158.6,157.20,156.8,141.4,139.3,136.4,129.0,127.8,127.5,127.0,126.7$, 123.5, 117.4, 60.9, 24.7, 14.0; MS (m/z) 317. 


\section{6-([1,1'-biphenyl]-4-yl)-2-methylnicotinohydrazide. (3)}

To a solution of $2(3 \mathrm{~g}, 9.54 \mathrm{mmol})$ in ethanol $(50 \mathrm{~mL})$, hydrazine hydrate $(99 \%, 2.5 \mathrm{~mL}, 47 \mathrm{mmol})$ was added dropwise. The reaction mixture was heated at reflux for $8 \mathrm{~h}$ then allowed to cool down to room temperature. The formed solid was separated by filtration and crystallized from ethanol to provide the desired product as white crystals $(2.73 \mathrm{~g}, 95 \%) \mathrm{mp}=95{ }^{\circ} \mathrm{C} ;{ }^{1} \mathrm{H}$ NMR $\left(400 \mathrm{MHz}\right.$, DMSO-d $\left.\mathrm{d}_{6}\right) \delta 9.61$ (brs, $1 \mathrm{H}), 8.22(\mathrm{~d}, J=8.4 \mathrm{~Hz}, 2 \mathrm{H}) .7 .90(\mathrm{~d}, J=8.3 \mathrm{~Hz}, 1 \mathrm{H}), 7.81(\mathrm{~d}, J=8.3 \mathrm{~Hz}, 2 \mathrm{H}), 7.79$ $(\mathrm{d}, J=8.3 \mathrm{~Hz}, 1 \mathrm{H}), 7.75(\mathrm{~d}, J=7.2 \mathrm{~Hz}, 2 \mathrm{H}), 7.51(\mathrm{t}, J=7.2 \mathrm{~Hz}, 2 \mathrm{H}), 7.42(\mathrm{t}, J=7.2$ $\mathrm{Hz}, 1 \mathrm{H}), 4.53$ (brs, 2H), 2.62 (s, 3H) ; ${ }^{13} \mathrm{C}$ NMR (101 MHz, DMSO-d 6 ) $\delta 167.2,155.6$, 155.4, 155.3, 140.9, 139.4, 137.0, 136.4, 128.9, 127.8, 127.2, 127.0, 126.7, 117.0, 23.0; MS (m/z) 303.

Potassium 5-(6-([1,1'-biphenyl]-4-yl)-2-methylpyridin-3-yl)-1,3,4-oxadiazole-2thiolate (4)

Potassium hydroxide $(0.0 .35 \mathrm{~g}, 8.5 \mathrm{mmol})$ was added to a solution of $\mathbf{3}$ ( $2.5 \mathrm{~g}$, $8.5 \mathrm{mmol})$ in ethanol $(15 \mathrm{~mL})$, followed by drop-wise addition of carbon disulphide $(2.5 \mathrm{~mL}, 93.5 \mathrm{mmol})$ over $0.5 \mathrm{~h}$. The reaction mixture was stirred at room temperature for an additional $15 \mathrm{~min}$ and then heated to reflux until the evolution of hydrogen sulfide gas ceased. After completion of the reaction, as monitored by TLC, the obtained intermediate 4 was poured on cold water $(50 \mathrm{~mL})$, filtered, washed with water, dried and crystallized from ethanol to provide white crystals $(2.9 \mathrm{~g}, 89 \%) \mathrm{mp}>300{ }^{\circ} \mathrm{C} ;{ }^{1} \mathrm{H}$ NMR (400 MHz, DMSO-d $\left.{ }_{6}\right) \delta 8.29(\mathrm{~d}, J=8.4 \mathrm{~Hz}, 2 \mathrm{H}) .8 .25(\mathrm{~d}, J=8.4 \mathrm{~Hz}, 1 \mathrm{H}), 8.08$ $(\mathrm{d}, J=8.4 \mathrm{~Hz}, 1 \mathrm{H}), 7.85(\mathrm{~d}, J=8.4 \mathrm{~Hz}, 2 \mathrm{H}), 7.77(\mathrm{~d}, J=6.8 \mathrm{~Hz}, 2 \mathrm{H}), 7.53(\mathrm{t}, J=7.2$ $\mathrm{Hz}, 2 \mathrm{H}), 7.43(\mathrm{t}, J=7.2 \mathrm{~Hz}, 1 \mathrm{H}), 2.85(\mathrm{~s}, 3 \mathrm{H}) ;{ }^{13} \mathrm{C} \mathrm{NMR}\left(101 \mathrm{MHz}, \mathrm{DMSO}-\mathrm{d}_{6}\right) \delta$ $164.5,156.8,155.6,155.1,141.9,139.7,137.3,136.8,129.5,127.9$, 127.5, 127.1, $126.9,118.1,116.9,25.3 ; \mathrm{MS}(\mathrm{m} / \mathrm{z}) 383$.

\section{2-(6-([1,1'-biphenyl]-4-yl)-2-methylpyridin-3-yl)-5-(methylthio)-1,3,4-oxadiazole} $\underline{(5)}$

The obtained white crystals $4(2.5 \mathrm{~g}, 6.5 \mathrm{mmol})$ was dissolved in water (30 $\mathrm{mL})$. Then, dimethyl sulfate $(1.55 \mathrm{~mL}, 12.4 \mathrm{mmol})$ was added dropwise with vigorous stirring. After $2 \mathrm{~h}$, the formed solid was filtered and washed with copious amounts of water to yield the title compound as a yellowish white solid $(2.1 \mathrm{~g}, 91 \%) ; \mathrm{mp}=138{ }^{\circ} \mathrm{C}$; ${ }^{1} \mathrm{HNMR}\left(400 \mathrm{MHz}, \mathrm{DMSO}-\mathrm{d}_{6}\right) \delta 8.30(\mathrm{~d}, \mathrm{~J}=8.4 \mathrm{~Hz}, 2 \mathrm{H}), 8.10(\mathrm{~d}, \mathrm{~J}=5.6 \mathrm{~Hz}, 1 \mathrm{H})$, $8.08(\mathrm{~d}, \mathrm{~J}=5.2 \mathrm{~Hz}, 1 \mathrm{H}), 7.85(\mathrm{~d}, \mathrm{~J}=8.4 \mathrm{~Hz}, 2 \mathrm{H}), 7.77(\mathrm{~d}, \mathrm{~J}=7.2 \mathrm{~Hz}, 2 \mathrm{H}), 7.52(\mathrm{t}, \mathrm{J}=$ $7.2 \mathrm{~Hz}, 2 \mathrm{H}), 7.43(\mathrm{t}, \mathrm{J}=7.6 \mathrm{~Hz}, 1 \mathrm{H}), 2.91(\mathrm{~s}, 3 \mathrm{H}), 2.80(\mathrm{~s}, 3 \mathrm{H}) ; 13 \mathrm{C}$ NMR $(101 \mathrm{MHz}$, DMSO-d $\left._{6}\right) \delta 164.2,156.4,155.8,155.2,141.4,139.3,136.3,135.6,129.0,127.9$, $127.4,127.1,126.7,117.7,116.7,36.3,25.1 ; \mathrm{MS}(\mathrm{m} / \mathrm{z}) 359$.

\section{$\underline{\text { 2-(6-([1,1'-biphenyl]-4-yl)-2-methylpyridin-3-yl)-5-(methylsulfonyl)-1,3,4- }}$ oxadiazole (6)}

To a solution of $5(2 \mathrm{~g}, 5.2 \mathrm{mmol})$ in dry DCM $(20 \mathrm{~mL})$, m-CPBA $(2.05 \mathrm{~g}, 11.6$ mmol) diluted with DCM $(10 \mathrm{~mL})$ was added portion wise with continuous stirring. Afterward, the reaction mixture was kept at $23{ }^{\circ} \mathrm{C}$ for $16 \mathrm{~h}$, additional DCM (10 mL) 
was added and the reaction mixture was washed with $25 \mathrm{~mL}$ of $5 \%$ aqueous solution of sodium metabisulfite, and $25 \mathrm{~mL}$ of $5 \%$ aqueous sodium carbonate. The organic layer was separated, dried and concentrated under reduced pressure to give the desired product as yellow crystals $(1.9 \mathrm{~g}, 93 \%) \mathrm{mp}=166{ }^{\circ} \mathrm{C} ;{ }^{1} \mathrm{H}$ NMR $(400 \mathrm{MHz}$, DMSO-d 6 ) $\delta 8.45(\mathrm{~d}, \mathrm{~J}=8.4 \mathrm{~Hz}, 1 \mathrm{H}), 8.32(\mathrm{~d}, J=8.4 \mathrm{~Hz}, 2 \mathrm{H}), 8.14(\mathrm{~d}, J=8.4 \mathrm{~Hz}, 1 \mathrm{H}), 7.86(\mathrm{~d}, J$ $=8.4 \mathrm{~Hz}, 2 \mathrm{H}), 7.77(\mathrm{~d}, J=7.6 \mathrm{~Hz}, 2 \mathrm{H}), 7.52(\mathrm{t}, J=7.6 \mathrm{~Hz}, 2 \mathrm{H}), 7.43(\mathrm{t}, J=7.6 \mathrm{~Hz}$, $1 \mathrm{H}), 3.75$ (s, 3H), 2.96 (s, 3H); ${ }^{13} \mathrm{C}$ NMR (101 MHz, DMSO-d 6 ) $\delta$ 165.6, 157.9, 157.6, 157.2, 142.1, 139.7, 138.6, 136.6, 129.5, 128.2, 128.0, 127.5, 127.1, 118.2, 116.8, 43.4, 25.5; MS (m/z) 391.

\section{Compounds 7 -24.}

General procedure: To a solution of $6(0.1 \mathrm{~g}, 0.25 \mathrm{mmol})$ in dry DMF $(5 \mathrm{~mL})$, appropriate amine, hydrazine, guanidine or carboximidate $(0.4 \mathrm{mmol})$ was added. The reaction mixture was heated at $80{ }^{\circ} \mathrm{C}$ for $0.5-12 \mathrm{~h}$, and then poured over ice water $(50$ $\mathrm{mL})$. The formed solid was extracted with ethyl acetate $(10 \mathrm{~mL})$. The organic layer was evaporated under reduced pressure. The obtained crude material was then purified by crystallization or column chromatography. Physical properties and spectral analysis of isolated products are listed below:

\section{5-(6-([1,1'-biphenyl]-4-yl)-2-methylpyridin-3-yl)- $N$-methyl-1,3,4-oxadiazol-2- amine. (7)}

White solid $(0.09 \mathrm{~g}, 93 \%) \mathrm{mp}=196{ }^{\circ} \mathrm{C} ;{ }^{1} \mathrm{H}$ NMR $\left(400 \mathrm{MHz}, \mathrm{DMSO}-\mathrm{d}_{6}\right) \delta 8.26$ $(\mathrm{d}, \mathrm{J}=8.3 \mathrm{~Hz}, 2 \mathrm{H}), 8.14(\mathrm{~d}, J=8.3 \mathrm{~Hz}, 1 \mathrm{H}), 8.07$ (brs, $1 \mathrm{H}), 8.02(\mathrm{~d}, J=8.3 \mathrm{~Hz}, 1 \mathrm{H})$, $7.83(\mathrm{~d}, J=8.3 \mathrm{~Hz}, 2 \mathrm{H}), 7.76(\mathrm{~d}, J=7.2 \mathrm{~Hz}, 2 \mathrm{H}), 7.52(\mathrm{t}, J=7.6 \mathrm{~Hz}, 2 \mathrm{H}), 7.42(\mathrm{t}, J=$ $7.6 \mathrm{~Hz}, 1 \mathrm{H}), 2.88(\mathrm{~s}, 3 \mathrm{H}), 2.80(\mathrm{~s}, 3 \mathrm{H}) ;{ }^{13} \mathrm{C}$ NMR (101 MHz, DMSO-d 6$) \delta 164.1$, $156.4,155.6,155.1,141.1,139.3,136.5,135.9,129.0,127.7,127.5,127.0,126.7$, 117.7, 117.4, 29.2, 25.2; MS (m/z) 342.

\section{5-(6-([1,1'-iphenyl]-4-yl)-2-methylpyridin-3-yl)- $N$-ethyl-1,3,4-oxadiazol-2-amine.}

$\underline{(8)}$

Yellowish white solid $(0.09 \mathrm{~g}, 93 \%) \mathrm{mp}=147{ }^{\circ} \mathrm{C} ;{ }^{1} \mathrm{H}$ NMR $(400 \mathrm{MHz}$, DMSO-d $\left.{ }_{6}\right) \delta 8.26(\mathrm{~d}, J=8.0 \mathrm{~Hz}, 2 \mathrm{H}), 8.14(\mathrm{~d}, J=8.0 \mathrm{~Hz}, 1 \mathrm{H}), 8.07(\mathrm{brs}, 1 \mathrm{H}), 8.03(\mathrm{~d}$, $J=8.0 \mathrm{~Hz}, 1 \mathrm{H}), 7.83(\mathrm{~d}, J=8.0 \mathrm{~Hz}, 2 \mathrm{H}), 7.76(\mathrm{~d}, J=7.6 \mathrm{~Hz}, 2 \mathrm{H}), 7.52(\mathrm{t}, J=7.6 \mathrm{~Hz}$, $2 \mathrm{H}), 7.42(\mathrm{t}, J=7.6 \mathrm{~Hz}, 1 \mathrm{H}), 3.32(\mathrm{q}, J=7.2,6.5 \mathrm{~Hz}, 2 \mathrm{H}), 2.88(\mathrm{~s}, 3 \mathrm{H}), 1.23(\mathrm{t}, J=7.2$ $\mathrm{Hz}, 3 \mathrm{H}) ;{ }^{13} \mathrm{C}$ NMR (101 MHz, DMSO-d 6 ) $\delta 163.4,156.4,155.5,155.1,141.1,139.3$, $136.3,135.8,129.0,127.8,127.5,127.2,126.7,117.8,117.2,37.54,25.2,14.6$; MS $(\mathrm{m} / \mathrm{z}) 356$.

\section{5-(6-([1,1'-biphenyl]-4-yl)-2-methylpyridin-3-yl)- $N$-butyl-1,3,4-oxadiazol-2-amine.} $\underline{(9)}$

Brown solid $(0.09 \mathrm{~g}, 93 \%) \mathrm{mp}=150{ }^{\circ} \mathrm{C} ;{ }^{1} \mathrm{H}$ NMR $\left(400 \mathrm{MHz}, \mathrm{DMSO}-\mathrm{d}_{6}\right) \delta$ $8.2^{\vee}(\mathrm{d}, J=8.3 \mathrm{~Hz}, 2 \mathrm{H}), 8.15(\mathrm{~d}, J=8.3 \mathrm{~Hz}, 1 \mathrm{H}), 8.09$ (brs, $\left.1 \mathrm{H}\right), 8.04(\mathrm{~d}, J=8.3 \mathrm{~Hz}$, $1 \mathrm{H}), 7.84(\mathrm{~d}, J=8.2 \mathrm{~Hz}, 2 \mathrm{H}), 7.77(\mathrm{~d}, J=7.2 \mathrm{~Hz}, 2 \mathrm{H}), 7.53(\mathrm{t}, J=7.3 \mathrm{~Hz}, 2 \mathrm{H}), 7.43(\mathrm{t}$, $J=7.3 \mathrm{~Hz}, 1 \mathrm{H}), 3.30(\mathrm{t}, J=7.2 \mathrm{~Hz}, 1 \mathrm{H}), 2.89(\mathrm{~s}, 3 \mathrm{H}), 1.61-155(\mathrm{~m}, 2 \mathrm{H}), 1.43-1.33(\mathrm{~m}$, 
2H), $0.94(\mathrm{t}, J=7.4 \mathrm{~Hz}, 2 \mathrm{H}) ;{ }^{13} \mathrm{C}$ NMR (101 MHz, DMSO-d 6 ) $\delta 164,156.9,156$, $155.4,141.5,139.9,137.5,136.2,129.4,127.9$, 127.6, 127.2, 126.9, 117.98, 117.3, 42.6, 25.6, 19.6, 14.4, 14.04.; MS (m/z) 384.

\section{5-(6-([1,1'-biphenyl]-4-yl)-2-methylpyridin-3-yl)- $N$-isopropyl-1,3,4-oxadiazol-2-} amine (10)

Yellow solid $(0.08 \mathrm{~g}, 85 \%) \mathrm{mp}=199{ }^{\circ} \mathrm{C} ;{ }^{1} \mathrm{H}$ NMR $\left(400 \mathrm{MHz}, \mathrm{DMSO}-\mathrm{d}_{6}\right) \delta$ $8.27(\mathrm{~d}, J=8.3 \mathrm{~Hz}, 2 \mathrm{H}), 8.14(\mathrm{~d}, J=8.3 \mathrm{~Hz}, 1 \mathrm{H}), 8.08$ (brs, $1 \mathrm{H}), 8.04(\mathrm{~d}, J=8.3 \mathrm{~Hz}$, $1 \mathrm{H}), 7.84(\mathrm{~d}, J=8.3 \mathrm{~Hz}, 2 \mathrm{H}), 7.76(\mathrm{~d}, J=8.3 \mathrm{~Hz}, 2 \mathrm{H}), 7.52(\mathrm{t}, J=7.3 \mathrm{~Hz}, 2 \mathrm{H}), 7.42(\mathrm{t}$, $J=7.3 \mathrm{~Hz}, 1 \mathrm{H}), 3.82-3.74(\mathrm{~m}, 1 \mathrm{H}), 2.88(\mathrm{~s}, 3 \mathrm{H}), 1.25(\mathrm{~m}, 6 \mathrm{H}) ;{ }^{13} \mathrm{C}$ NMR $(101 \mathrm{MHz}$, DMSO-d $\left._{6}\right) \delta 164.1,156.2,155.5,155.1,141.1,139.3,136.6,135.8,129.0,127.2$, 127.0, 127, 126.6, 117.8, 117.6, 44.9, 25.1, 14.3; MS (m/z) 370.

\section{5-(6-([1,1'-biphenyl]-4-yl)-2-methylpyridin-3-yl)- $N$-cyclopropyl-1,3,4-0xadiazol-2-} amine. (11)

White solid $(0.09 \mathrm{~g}, 90 \%) \mathrm{mp}=200{ }^{\circ} \mathrm{C} ;{ }^{1} \mathrm{H}$ NMR $\left(400 \mathrm{MHz}, \mathrm{DMSO}-\mathrm{d}_{6}\right) \delta 8.26$ $(\mathrm{d}, J=8.3 \mathrm{~Hz}, 2 \mathrm{H}), 8.18(\mathrm{~d}, J=8.3 \mathrm{~Hz}, 1 \mathrm{H}), 8.12(\mathrm{brs}, 1 \mathrm{H}), 8.03(\mathrm{~d}, J=8.3 \mathrm{~Hz}, 1 \mathrm{H})$, $7.83(\mathrm{~d}, J=8.3 \mathrm{~Hz}, 2 \mathrm{H}), 7.76(\mathrm{~d}, J=7.3 \mathrm{~Hz} 2 \mathrm{H}), 7.51(\mathrm{t}, J=7.3 \mathrm{~Hz}, 2 \mathrm{H}), 7.42(\mathrm{t}, J=$ $8.3 \mathrm{~Hz}, 1 \mathrm{H}), 2.79(\mathrm{~s}, 3 \mathrm{H}), 2.73-2.66(\mathrm{~m}, 1 \mathrm{H}), 0.76-0.71(\mathrm{~m}, 2 \mathrm{H}), 0.60-0.56(\mathrm{~m}$, $2 \mathrm{H}) ;{ }^{13} \mathrm{C}$ NMR (101 MHz, DMSO-d 6 ) $\delta 164,156.8,155.6,155.2,141.1,139.3,136.5$, $135.9,129,127.4,127.2,127,126.6,117.7,117.6,25.1,24.2,14.3 ; \mathrm{MS}(\mathrm{m} / \mathrm{z}) 368$.

\section{5-(6-([1,1'-biphenyl]-4-yl)-2-methylpyridin-3-yl)- $N$-cyclopentyl-1,3,4-oxadiazol-2-} amine. (12)

White solid $(0.09 \mathrm{~g}, 90 \%) \mathrm{mp}=200{ }^{\circ} \mathrm{C} ;{ }^{1} \mathrm{H}$ NMR $\left(400 \mathrm{MHz}, \mathrm{DMSO}-\mathrm{d}_{6}\right) \delta 8.30$ (d, $J=8.4 \mathrm{~Hz}, 2 \mathrm{H}), 8.15(\mathrm{~d}, J=8.4 \mathrm{~Hz}, 1 \mathrm{H}), 8.09$ (brs, $1 \mathrm{H}), 8.05(\mathrm{~d}, J=8.4 \mathrm{~Hz}, 1 \mathrm{H})$, $7.86(\mathrm{~d}, J=8.2 \mathrm{~Hz}, 2 \mathrm{H}), 7.77(\mathrm{~d}, J=7.2 \mathrm{~Hz}, 2 \mathrm{H}), 7.53(\mathrm{t}, J=7.3 \mathrm{~Hz}, 2 \mathrm{H}), 7.43(\mathrm{t}, J=$ $7.2 \mathrm{~Hz}, 1 \mathrm{H}), 4.00-3.92(\mathrm{~m}, 1 \mathrm{H}), 2.80(\mathrm{~s}, 3 \mathrm{H}), 1.96-1.93(\mathrm{~m}, 2 \mathrm{H}), 1.75-1.61(\mathrm{~m}, 2 \mathrm{H})$, $1.59-1.55(\mathrm{~m}, 4 \mathrm{H}) ;{ }^{13} \mathrm{C}$ NMR $\left(101 \mathrm{MHz}, \mathrm{DMSO}_{6}\right) \delta 164.4,156.8,155.8,155.4$, $141.9,139.7,136.8,134.9,129.5,127.9,127.5,127.1,127.1,126.9,117.4,117.3,32.6$, 25.5, 24.1, 23.7, 14.7; MS (m/z) 396.

\section{5-(6-([1,1'-biphenyl]-4-yl)-2-methylpyridin-3-yl)- $N$-cyclohexyl-1,3,4-oxadiazol-2- amine. (13).}

Black solid $(0.09 \mathrm{~g}, 90 \%) \mathrm{mp}=183{ }^{\circ} \mathrm{C} ;{ }^{1} \mathrm{H}$ NMR $(400 \mathrm{MHz}$, DMSO-d 6 ) $\delta 8.26$ $(\mathrm{d}, J=8.4 \mathrm{~Hz}, 2 \mathrm{H}), 8.14(\mathrm{~d}, J=8.4 \mathrm{~Hz}, 1 \mathrm{H}), 8.08$ (brs, $1 \mathrm{H}), 8.03(\mathrm{~d}, J=8.4 \mathrm{~Hz}, 1 \mathrm{H})$, $7.83(\mathrm{~d}, J=8.4 \mathrm{~Hz}, 2 \mathrm{H}), 7.76(\mathrm{~d}, J=7.2 \mathrm{~Hz}, 2 \mathrm{H}), 7.52(\mathrm{t}, J=7.2 \mathrm{~Hz}, 2 \mathrm{H}), 7.42(\mathrm{t}, J=$ $7.2 \mathrm{~Hz}, 1 \mathrm{H}), 3.46-3.32(\mathrm{~m}, 1 \mathrm{H}), 2.88(\mathrm{~s}, 3 \mathrm{H}), 2.02-1.97(\mathrm{~m}, 2 \mathrm{H}), 1.75-1.73(\mathrm{~m}, 2 \mathrm{H})$, 1.60-1.54 (m, 2H), 1.32-1.27 (m, 4H); ${ }^{13} \mathrm{C}$ NMR (101 MHz, DMSO-d 6 ) $\delta 162.8156 .2$, 155.5, 141.1, 139.3, 136.6, 135.8, 129.0, 127.5, 127.3, 127.1, 126.7, 117.6, 116.8, 51.8, 32.3, 25.2, 24.4, 14.3; MS $(\mathrm{m} / \mathrm{z}) 410$. 
$N^{1}$-(5-(6-([1,1'-biphenyl]-4-yl)-2-methylpyridin-3-yl)-1,3,4-oxadiazol-2-yl)- $N^{2}, N^{2}-$ dimethylethane -1,2-diamine. (14)

Gray solid $(0.06 \mathrm{~g}, 65 \%) \mathrm{mp}=165{ }^{\circ} \mathrm{C} ;{ }^{1} \mathrm{H}$ NMR $\left(400 \mathrm{MHz}, \mathrm{DMSO}-\mathrm{d}_{6}\right) \delta 8.27$ $(\mathrm{d}, J=8.4 \mathrm{~Hz}, 2 \mathrm{H}), 8.16(\mathrm{~d}, J=8.4 \mathrm{~Hz}, 1 \mathrm{H}), 8.09(\mathrm{brs}, 1 \mathrm{H}), 8.04(\mathrm{~d}, J=8 \mathrm{~Hz}, 1 \mathrm{H})$, $7.84(\mathrm{~d}, J=8.4 \mathrm{~Hz}, 2 \mathrm{H}), 7.77(\mathrm{~d}, J=6.8 \mathrm{~Hz}, 2 \mathrm{H}), 7.52(\mathrm{t}, J=7.2 \mathrm{~Hz}, 2 \mathrm{H}), 7.43(\mathrm{t}, J=$ $7.2 \mathrm{~Hz}, 1 \mathrm{H}), 3.63(\mathrm{t}, J=6.4 \mathrm{~Hz}, 2 \mathrm{H}), 2.80(\mathrm{~s}, 3 \mathrm{H}), 2.48(\mathrm{t}, J=6.4 \mathrm{~Hz}, 2 \mathrm{H}), 2.20(\mathrm{~s}, 6 \mathrm{H})$; ${ }^{13} \mathrm{C}$ NMR (101 MHz, DMSO-d $\left.\mathrm{d}_{6}\right) \delta 164.0,156.8,155.8,155.4,141.6,139.8,136.2$, $135.5,129.4,127.9,127.5,127.1,126.8,117.3,117.2,45.5,37.5,30.7,25.7$; MS $(\mathrm{m} / \mathrm{z})$ 399.

\section{2-((5-(6-([1,1'-biphenyl]-4-yl)-2-methylpyridin-3-yl)-1,3,4-oxadiazol-2-} yl)amino)ethan-1-ol. (15)

Yellow solid $(0.09 \mathrm{~g}, 90 \%) \mathrm{mp}=155{ }^{\circ} \mathrm{C} ;{ }^{1} \mathrm{H}$ NMR $\left(400 \mathrm{MHz}, \mathrm{DMSO}-\mathrm{d}_{6}\right) \delta 8.26(\mathrm{~d}$, $J=8.4 \mathrm{~Hz}, 2 \mathrm{H}), 8.14(\mathrm{~d}, J=8.4 \mathrm{~Hz}, 1 \mathrm{H}), 8.07$ (brs, $1 \mathrm{H}), 8.03(\mathrm{~d}, J=8.4 \mathrm{~Hz}, 1 \mathrm{H}), 7.83$ $(\mathrm{d}, J=8.4 \mathrm{~Hz} 2 \mathrm{H}), 7.76(\mathrm{~d}, J=8.4 \mathrm{~Hz}, 2 \mathrm{H}), 7.52(\mathrm{t}, J=7.2 \mathrm{~Hz}, 2 \mathrm{H}), 7.42(\mathrm{t}, J=7.2 \mathrm{~Hz}$, $1 \mathrm{H}), 4.82(\mathrm{brs}, 1 \mathrm{H}), 3.59(\mathrm{t}, J=3.6 \mathrm{~Hz}, 2 \mathrm{H}), 2.79(\mathrm{~s}, 3 \mathrm{H}), 2.63-2.62(\mathrm{~m}, 2 \mathrm{H}) ;{ }^{13} \mathrm{C} \mathrm{NMR}$ $\left(101 \mathrm{MHz}, \mathrm{DMSO}-\mathrm{d}_{6}\right) \delta 163.6,156.3,155.5,155.1,141.1,139.3,136.6,135.8,129.0$, 127.8, 127.4, 127.2, 126.6, 117.8, 117.6, 59.3, 45.3, 25.2; MS (m/z) 372.

\section{5-(6-([1,1'-biphenyl]-4-yl)-2-methylpyridin-3-yl)- $N, N$-dimethyl-1,3,4-oxadiazol-2- amine. (16)}

Yellow solid $(0.09 \mathrm{~g}, 90 \%) \mathrm{mp}=179{ }^{\circ} \mathrm{C} ;{ }^{1} \mathrm{H}$ NMR $\left(400 \mathrm{MHz}, \mathrm{DMSO}-\mathrm{d}_{6}\right) \delta 8.27(\mathrm{~d}$, $J=8.8 \mathrm{~Hz}, 2 \mathrm{H}), 8.08(\mathrm{~d}, J=8.4 \mathrm{~Hz}, 1 \mathrm{H}), 8.03(\mathrm{~d}, J=8.4 \mathrm{~Hz}, 1 \mathrm{H}), 7.83(\mathrm{~d}, J=8.0 \mathrm{~Hz}$, 2H), $7.76(\mathrm{~d}, J=7.6 \mathrm{~Hz}, 2 \mathrm{H}), 7.52(\mathrm{t}, J=7.6 \mathrm{~Hz}, 2 \mathrm{H}), 7.42(\mathrm{t}, J=7.6 \mathrm{~Hz}, 1 \mathrm{H}), 3.13(\mathrm{~s}$, $6 \mathrm{H}), 2.80(\mathrm{~s}, 3 \mathrm{H}) ;{ }^{13} \mathrm{C}$ NMR $\left(101 \mathrm{MHz}, \mathrm{DMSO}_{-}\right) \delta 164.1,156.4,155.5,155.1,141.5$, $139.4,136.6,135.9,129.0,127.5,127.3,127.1,126.7,117.7,117.6,37.6,25.5$; MS $(\mathrm{m} / \mathrm{z}) 356 \mathrm{M}^{+}$.

\section{5-(6-([1,1'-biphenyl]-4-yl)-2-methylpyridin-3-yl)- $N, N$-diethyl-1,3,4-oxadiazol-2- amine. (17)}

Brown solid $(0.08 \mathrm{~g}, 87 \%) \mathrm{mp}=112{ }^{\circ} \mathrm{C} ;{ }^{1} \mathrm{H}$ NMR $\left(400 \mathrm{MHz}, \mathrm{DMSO}-\mathrm{d}_{6}\right) \delta 8.27(\mathrm{~d}, J=$ $8.8 \mathrm{~Hz}, 2 \mathrm{H}), 8.07(\mathrm{~d}, J=8.4 \mathrm{~Hz}, 1 \mathrm{H}), 8.02(\mathrm{~d}, J=8.4 \mathrm{~Hz}, 1 \mathrm{H}), 7.83(\mathrm{~d}, J=8.4 \mathrm{~Hz}, 2 \mathrm{H})$, $7.77(\mathrm{~d}, J=8.4 \mathrm{~Hz}, 2 \mathrm{H}), 7.52(\mathrm{t}, J=7.6 \mathrm{~Hz}, 2 \mathrm{H}), 7.42(\mathrm{t}, J=7.6 \mathrm{~Hz}, 1 \mathrm{H}), 3.53(\mathrm{q}, J=$ $7.2 \mathrm{~Hz}, 4 \mathrm{H}), 2.80(\mathrm{~s}, 3 \mathrm{H}), 1.24(\mathrm{t}, J=7.2 \mathrm{~Hz}, 6 \mathrm{H}) ;{ }^{13} \mathrm{C}$ NMR $(101 \mathrm{MHz}$, DMSO-d 6 ) $\delta$ $164.6,156.9$, 155.8, 155.5, 141.9, 139.8, 137.7, 136.3, 129.4, 127.9, 127.7, 127.5, $126.9,117.6,117.3,43.4,25.6,13.5 ; \mathrm{MS}(\mathrm{m} / \mathrm{z}) 384$.

2-(6-([1,1'-biphenyl]-4-yl)-2-methylpyridin-3-yl)-5-(azetidin-1-yl)-1,3,4-oxadiazole. $\underline{(18)}$

White solid $(0.08 \mathrm{~g}, 85 \%) \mathrm{mp}=152{ }^{\circ} \mathrm{C} ;{ }^{1} \mathrm{H}$ NMR $\left(400 \mathrm{MHz}, \mathrm{DMSO}-\mathrm{d}_{6}\right) \delta 8.29(\mathrm{~d}, J$ $=8.4 \mathrm{~Hz}, 2 \mathrm{H}), 8.12(\mathrm{~d}, J=8.0 \mathrm{~Hz}, 1 \mathrm{H}), 8.08(\mathrm{~d}, J=8.4 \mathrm{~Hz}, 1 \mathrm{H}), 7.84(\mathrm{~d}, J=8.4 \mathrm{~Hz}$, 2H), $7.77(\mathrm{~d}, J=7.3,1.9 \mathrm{~Hz}, 2 \mathrm{H}), 7.52(\mathrm{t}, J=7.3 \mathrm{~Hz}, 2 \mathrm{H}), 7.43(\mathrm{t}, J=7.2 \mathrm{~Hz}, 1 \mathrm{H}), 3.55$ $(\mathrm{t}, J=5.2 \mathrm{~Hz}, 4 \mathrm{H}), 2.80(\mathrm{~s}, 3 \mathrm{H}), 2.31(\mathrm{~m}, 2 \mathrm{H}) ;{ }^{13} \mathrm{C}$ NMR $\left(101 \mathrm{MHz}, \mathrm{DMSO}-\mathrm{d}_{6}\right) \delta$ 
$164.6,156.9,155.9,141.8,139.8,136.3,135.9,129.4,127.9,127.5,127.1,126.9$, $117.9,117.3,45.9,25.7,14.6 ; \mathrm{MS}(\mathrm{m} / \mathrm{z}) 368$.

$\underline{\text { 2-(6-([1,1'-biphenyl]-4-yl)-2-methylpyridin-3-yl)-5-(pyrrolidin-1-yl)-1,3,4- }}$ oxadiazole. (19)

White solid $(0.08 \mathrm{~g}, 85 \%) \mathrm{mp}=152{ }^{\circ} \mathrm{C} ;{ }^{1} \mathrm{H}$ NMR $(400 \mathrm{MHz}$, DMSO-d 6 ) $\delta 8.27(\mathrm{~d}, J$ $=8.4 \mathrm{~Hz}, 2 \mathrm{H}), 8.08(\mathrm{~d}, J=8.0 \mathrm{~Hz}, 1 \mathrm{H}), 8.03(\mathrm{~d}, J=8.4 \mathrm{~Hz}, 1 \mathrm{H}), 7.85(\mathrm{~d}, J=8.4 \mathrm{~Hz}$, 2H), $7.76(\mathrm{~d}, J=7.2 \mathrm{~Hz}, 2 \mathrm{H}), 7.52(\mathrm{t}, J=7.6 \mathrm{~Hz}, 2 \mathrm{H}), 7.48(\mathrm{t}, J=7.2 \mathrm{~Hz}, 1 \mathrm{H}), 3.53(\mathrm{t}, J$ $=6.8 \mathrm{~Hz}, 4 \mathrm{H}), 2.80(\mathrm{~s}, 3 \mathrm{H}), 1.96(\mathrm{t}, J=6.8 \mathrm{~Hz}, 4 \mathrm{H}) ;{ }^{13} \mathrm{C}$ NMR $\left(101 \mathrm{MHz}, \mathrm{DMSO}_{-} \mathrm{d}_{6}\right) \delta$ $164.1,156.4$, 155.5, 155.1, 141.4, 139.3, 136.3, 135.9, 129.0, 127.9, 127.5, 127.0, 126.7, 117.8, 117.4, 47.5, 25.1, 14.3; MS (m/z) 382.

$\underline{\text { 2-(6-([1,1'-biphenyl]-4-yl)-2-methylpyridin-3-yl)-5-(piperidin-1-yl)-1,3,4- }}$ oxadiazole. (20)

Buff solid $(0.08 \mathrm{~g}, 85 \%) \mathrm{mp}=164{ }^{\circ} \mathrm{C} ;{ }^{1} \mathrm{H}$ NMR $\left(400 \mathrm{MHz}, \mathrm{DMSO}-\mathrm{d}_{6}\right) \delta 8.28(\mathrm{~d}, J=$ $8.4 \mathrm{~Hz}, 2 \mathrm{H}), 8.10(\mathrm{~d}, J=8.4 \mathrm{~Hz}, 1 \mathrm{H}), 8.04(\mathrm{~d}, J=8.4 \mathrm{~Hz}, 1 \mathrm{H}), 7.84(\mathrm{~d}, J=8.4 \mathrm{~Hz}, 2 \mathrm{H})$, $7.77(\mathrm{~d}, J=7.2 \mathrm{~Hz}, 2 \mathrm{H}), 7.53(\mathrm{t}, J=7.2 \mathrm{~Hz}, 2 \mathrm{H}), 7.43(\mathrm{t}, J=7.2 \mathrm{~Hz}, 1 \mathrm{H}), 3.53-3.3(\mathrm{~m}$, $4 \mathrm{H}), 2.81(\mathrm{~s}, 3 \mathrm{H}), 1.63-1.49(\mathrm{~m}, 4 \mathrm{H}), 1.23-1.19(\mathrm{~m}, 2 \mathrm{H}) ;{ }^{13} \mathrm{C}$ NMR (101 MHz, DMSO$\left.\mathrm{d}_{6}\right) \delta 164.3,156.8,155.6,155.4,141.7,139.8,136.8,135.8,129.5,127.7,127.5,127.1$, 126.9, 117.8, 117.7, 47.1, 25.7, 23.6, 14.6; MS (m/z) 396.

$\underline{\text { 1-(5-(6-([1,1'-biphenyl]-4-yl)-2-methylpyridin-3-yl)-1,3,4-oxadiazol-2-yl)azetidin-3- }}$ $\underline{\text { ol. }(21)}$

White solid $(0.08 \mathrm{~g}, 85 \%) \mathrm{mp}=205{ }^{\circ} \mathrm{C} ;{ }^{1} \mathrm{H}$ NMR $(400 \mathrm{MHz}$, DMSO-d 6 ) $\delta 8.27(\mathrm{~d}, J$ $=8.4 \mathrm{~Hz}, 2 \mathrm{H}), 8.08(\mathrm{~d}, J=8.4 \mathrm{~Hz}, 1 \mathrm{H}), 8.03(\mathrm{~d}, J=8.0 \mathrm{~Hz}, 1 \mathrm{H}), 7.83(\mathrm{~d}, J=8.4 \mathrm{~Hz}$, 2H), $7.76(\mathrm{~d}, J=7.6,2 \mathrm{H}), 7.52(\mathrm{t}, J=7.6 \mathrm{~Hz}, 2 \mathrm{H}), 7.42(\mathrm{t}, J=7.6 \mathrm{~Hz}, 1 \mathrm{H}), 5.94$ (brs, $1 \mathrm{H}), 4.69(\mathrm{p}, J=5.2 \mathrm{~Hz}, 1 \mathrm{H}), 4.39(\mathrm{dd}, J=4.4 \mathrm{~Hz}, J=9.2 \mathrm{~Hz}, 2 \mathrm{H}), 3.99(\mathrm{dd}, J=6.8$ $\mathrm{Hz}, J=9.2 \mathrm{~Hz}, 2 \mathrm{H}), 2.79(\mathrm{~s}, 3 \mathrm{H}) ;{ }^{13} \mathrm{C}$ NMR (101 MHz, DMSO-d $\left.{ }_{6}\right) \delta 164.1,156.5$, $155.8,155.5,141.2,139.2,136.6,135.4,129.1,127.4,127.3,127.0,126.6,117.6$, 117.5, 61.8, 42.4, 25.1; MS (m/z) 384 .

\section{4-(5-(6-([1,1'-biphenyl]-4-yl)-2-methylpyridin-3-yl)-1,3,4-oxadiazol-2-}

yl)morpholine .(22)

White solid $(0.08 \mathrm{~g}, 85 \%) \mathrm{mp}=200{ }^{\circ} \mathrm{C} ;{ }^{1} \mathrm{H}$ NMR $\left(400 \mathrm{MHz}, \mathrm{DMSO}_{\mathrm{d}}\right) \delta 8.28(\mathrm{~d}, J$ $=8.4 \mathrm{~Hz}, 2 \mathrm{H}), 8.12(\mathrm{~d}, J=8.4 \mathrm{~Hz}, 1 \mathrm{H}), 8.05(\mathrm{~d}, J=8.4 \mathrm{~Hz}, 1 \mathrm{H}), 7.84(\mathrm{~d}, J=8.4 \mathrm{~Hz}$, $2 \mathrm{H}), 7.76(\mathrm{~d}, J=7.2 \mathrm{~Hz}, 2 \mathrm{H}), 7.52(\mathrm{t}, J=7.6 \mathrm{~Hz}, 2 \mathrm{H}), 7.42(\mathrm{t}, J=7.2 \mathrm{~Hz}, 1 \mathrm{H}), 3.76(\mathrm{t}$, $J=5.2 \mathrm{~Hz}, 4 \mathrm{H}), 3.53(\mathrm{t}, J=5.2 \mathrm{~Hz}, 4 \mathrm{H}), 2.89(\mathrm{~s}, 3 \mathrm{H}) ;{ }^{13} \mathrm{C}$ NMR $(101 \mathrm{MHz}$, DMSO-d 6 ) $\delta 163.8,156.8,155.6,155.3,141.0,139.1,136.3,135.6,129.0,127.8,127.3,127.0$, 126.7, 117.7, 117.5, 65.2, 45.7, 25.21.; MS (m/z) 384 . 


\section{2-(6-([1,1'-biphenyl]-4-yl)-2-methylpyridin-3-yl)-5-(4-methylpiperazin-1-yl)-1,3,4-} oxadiazole. $(23)$

White solid $(0.08 \mathrm{~g}, 85 \%) \mathrm{mp}=200{ }^{\circ} \mathrm{C} ;{ }^{1} \mathrm{H}$ NMR $\left(400 \mathrm{MHz}, \mathrm{DMSO}-\mathrm{d}_{6}\right) \delta 8.26(\mathrm{~d}, J$ $=8.4 \mathrm{~Hz}, 2 \mathrm{H}), 8.07(\mathrm{~d}, J=8.0 \mathrm{~Hz}, 1 \mathrm{H}), 8.02(\mathrm{~d}, J=8.4 \mathrm{~Hz}, 1 \mathrm{H}), 7.82(\mathrm{~d}, J=8.4 \mathrm{~Hz}$, 2H), $7.75(\mathrm{~d}, J=7.2 \mathrm{~Hz}, 2 \mathrm{H}), 7.51(\mathrm{t}, J=7.6 \mathrm{~Hz}, 2 \mathrm{H}), 7.42(\mathrm{t}, J=7.2 \mathrm{~Hz}, 1 \mathrm{H}), 3.54(\mathrm{t}, J$ $=5.0 \mathrm{~Hz}, 4 \mathrm{H}), 2.79(\mathrm{~s}, 3 \mathrm{H}), 2.45(\mathrm{t}, J=5.0 \mathrm{~Hz}, 4 \mathrm{H}), 2.36(\mathrm{~s}, 3 \mathrm{H}) ;{ }^{13} \mathrm{C} \mathrm{NMR}(101 \mathrm{MHz}$, DMSO-d $\left._{6}\right) \delta 164.0,156.4,155.6,155.3,141.1,139.3,136.5,135.9,129.0,127.8,127.3$, 127.0, 126.70, 117.6, 117.5, 47.6, 47.5, 35.6, 25.2; MS $(\mathrm{m} / z) 411$.
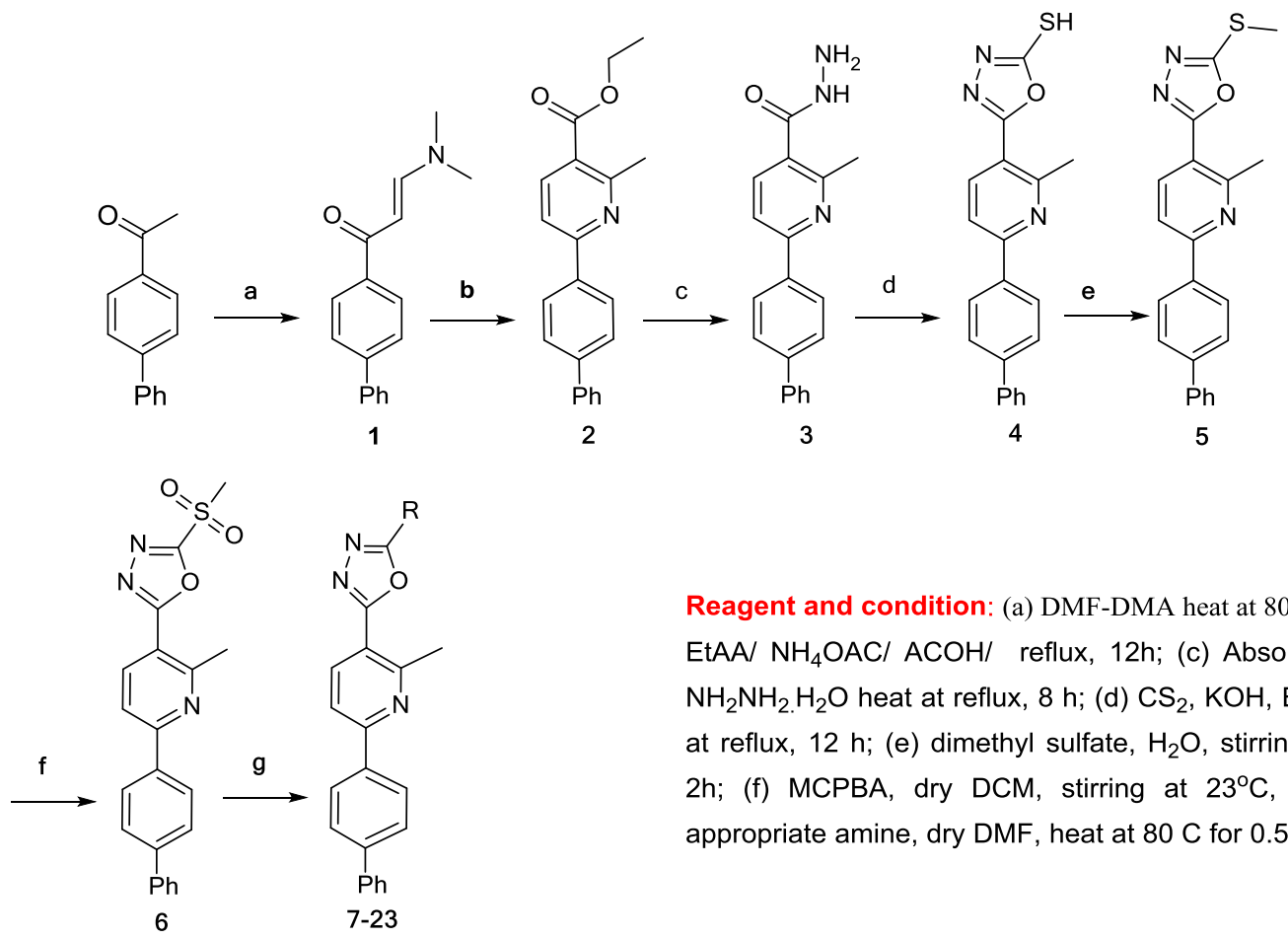

Reagent and condition: (a) DMF-DMA heat at 80 0C, 8h; (b) EtAA/ $\mathrm{NH}_{4} \mathrm{OAC} / \mathrm{ACOH} /$ reflux, 12h; (c) Absolute $\mathrm{EtOH}$, $\mathrm{NH}_{2} \mathrm{NH}_{2} \mathrm{H}_{2} \mathrm{O}$ heat at reflux, $8 \mathrm{~h}$; (d) $\mathrm{CS}_{2}, \mathrm{KOH}$, EtOH, heat at reflux, $12 \mathrm{~h}$; (e) dimethyl sulfate, $\mathrm{H}_{2} \mathrm{O}$, stirring at $23^{\circ} \mathrm{C}$, 2h; (f) MCPBA, dry DCM, stirring at $23^{\circ} \mathrm{C}, 16 \mathrm{~h}$; $(\mathrm{g})$ appropriate amine, dry DMF, heat at $80 \mathrm{C}$ for $0.5-8 \mathrm{~h}$.

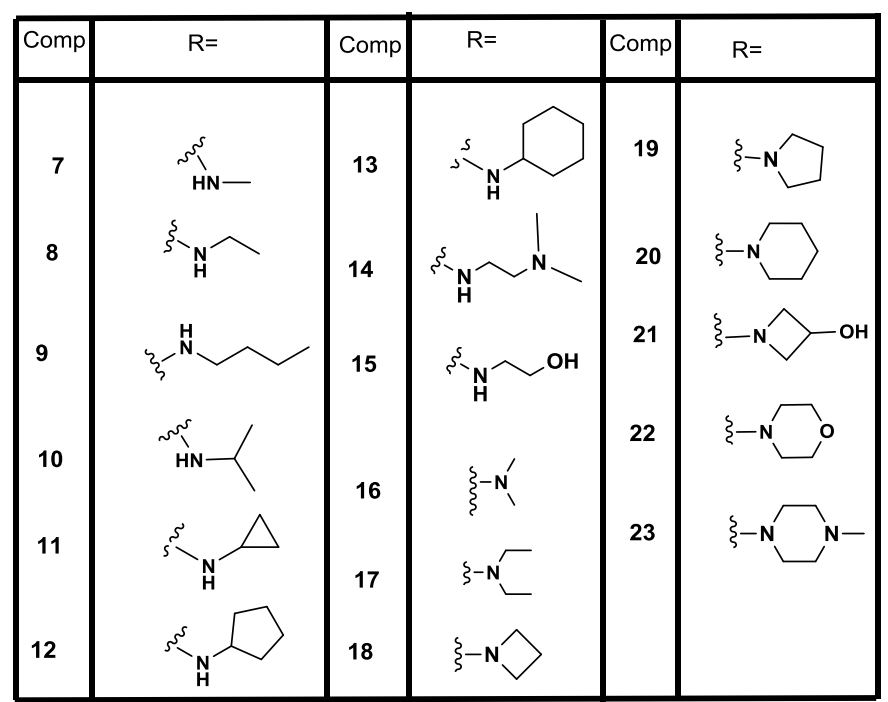




\section{III- Antimicrobial Activity}

\section{Antimicrobial investigation:}

The minimum inhibitory concentrations (MICs) of the tested compounds and control drugs; linezolid, vancomycin, gentamicin (antibiotics), azithromycin and 5fluorocytosine (5-FC) (antifungal drug) were determined using the broth microdilution method, according to guidelines outlined by the Clinical and Laboratory Standards Institute CLSI) (Clinical and Laboratory Standards Institute, 2007, 2008, 2012) or as described in previous reports (Geers \& Donabedian, 1989), with some modifications, against clinically-relevant bacterial (methicillin-resistant Staphylococcus aureus (MRSA), Escherichia coli, Clostridium difficile and Neisseria gonorrhea strains) and fungal (Candida albicans) strains. S. aureus and E. coli were grown aerobically overnight on tryptone soy agar plates at $37^{\circ} \mathrm{C}$. C. difficile was grown anaerobically on brain heart infusion supplemented agar at $37^{\circ} \mathrm{C}$ for 48 hours. N. gonorrhoea was grown on Brucella broth supplemented with yeast extract, neopeptone, hematin, pyridoxal and $\mathrm{NAD}$ at $37^{\circ} \mathrm{C}$ for 24 hours in presence of $5 \% \mathrm{CO}_{2}$. C.albicans was grown aerobically overnight on yeast peptone dextrose (YPD) agar plate at $35^{\circ} \mathrm{C}$. Afterwards, a bacterial solution equivalent to $0.5 \mathrm{McFarland}$ standard was prepared and diluted in cationadjusted Mueller-Hinton broth (CAMHB) (for S. aureus and E. coli) to achieve a bacterial concentration of about $5 \times 105 \mathrm{CFU} / \mathrm{mL}$. C. difficile was diluted in brain heart infusion supplemented broth, supplemented with yeast extract, hemin and vitamin $\mathrm{K}$ to achieve a bacterial concentration of about $5 \times 105 \mathrm{CFU} / \mathrm{mL}$. N. gonorrhoeae was diluted in Brucella broth supplemented with yeast extract, neopeptone, hematin, pyridoxal and NAD to achieve a bacterial concentration of about $1 \times 106 \mathrm{CFU} / \mathrm{mL}$. C. albicans was diluted in Roswell Park Memorial Institute (RPMI 1640) medium with glutamine and without bicarbonate (GIBCO by Life Technologies, Green Island, NY, USA) which was buffered to $\mathrm{pH} 7.0$ with $0.165 \mathrm{M}$ of [3-(N-morpholino)propanesulfonic acid] (MOPS) (dot scientific inc., Burton, MI, USA) to achieve a fungal concentration of about $1.5 \times$ $103 \mathrm{CFU} / \mathrm{mL}$. Compounds and control drugs were added in the first row of the 96-well plates and serially diluted with the corresponding media containing bacteria/fungi. Plates were then, incubated as previously described. MICs reported in Table (1) are the minimum concentration of the compounds and control drugs that could completely inhibit the visual growth of bacteria/fungi. 


\begin{tabular}{|c|c|c|c|c|}
\hline Code & $\begin{array}{c}\text { Methicillin-resistant } \\
\text { S. aureus } \\
\text { NRS384 } \\
\text { (MRSA USA300) }\end{array}$ & $\begin{array}{c}\text { Clostridium } \\
\text { difficile } \\
\text { ATCC BAA } \\
1870\end{array}$ & $\begin{array}{c}\text { Escherichia coli } \\
\text { JW55031 } \\
\text { (TolC Mutant) }\end{array}$ & $\begin{array}{c}\text { Candida } \\
\text { albicans } \\
\text { SS5314 } \\
\text { (wild-type) }\end{array}$ \\
\hline 7 & $>64$ & 64 & $>64$ & $>64$ \\
\hline 8 & $>64$ & $>64$ & $>64$ & $>64$ \\
\hline 9 & $>64$ & $>64$ & $>64$ & $>64$ \\
\hline 10 & $>64$ & $>64$ & $>64$ & $>64$ \\
\hline 11 & $>64$ & $>64$ & $>64$ & $>64$ \\
\hline 12 & $>64$ & 32 & $>64$ & $>64$ \\
\hline 13 & $>64$ & $>64$ & $>64$ & $>64$ \\
\hline 14 & 8 & 16 & 16 & 8 \\
\hline 15 & $>64$ & $>64$ & $>64$ & $>64$ \\
\hline 16 & 16 & 32 & $>64$ & 32 \\
\hline 17 & $>64$ & $>64$ & $>64$ & $>64$ \\
\hline 18 & 32 & $>64$ & $>64$ & 8 \\
\hline 19 & $>64$ & $>64$ & $>64$ & $>64$ \\
\hline 20 & $>64$ & $>64$ & $>64$ & $>64$ \\
\hline 21 & $>64$ & $>64$ & $>64$ & $>64$ \\
\hline 22 & $>64$ & $>64$ & $>64$ & $>64$ \\
\hline 23 & 8 & 8 & $>64$ & 8 \\
\hline Linezolid & 1 & $>64$ & 8 & NT \\
\hline
\end{tabular}

Table (1); Antimicrobial activities of compounds 7-23.

\section{IV-Conclusion}

From the above mentioned results in table (1) it was found that (for the On MRSA-US300 activity) the highest activity was obtained with compounds $\mathbf{1 4}$ and $\mathbf{2 3}$ with MIC value about $8 \mu \mathrm{g} / \mathrm{mL}$ followed by compound $\mathbf{1 6}$ with MIC value about 16 $\mu \mathrm{g} / \mathrm{mL}$. The other compounds show very low activity. On the other hand, most synthesized compounds gave very weak activity against Clostridium difficile ATCC BAA 1870 except compounds 23 which has moderate activity with MIC $8 \mu \mathrm{g} / \mathrm{mL}$. Compound $\mathbf{1 4}$ have weak activity but still active against $C$. difficile. This means that the presence of terminal hydrogen bond acceptor group is essential for activity against resistant gram positive bacteria. For the activity against gram negative tested Escherichia coli JW55031 (TolC Mutant) all newly synthesized compounds shows no activity except compound $\mathbf{1 4}$ which showed weak activity with MIAC $16 \mu \mathrm{g} / \mathrm{Ml}$. In the other had; the antifungal activity against Candida albicans SS5314 (wild-type) also compounds 14,18 and 23 showed moderate activity with MIC value equal $8 \mu \mathrm{g} / \mathrm{mL}$.

As a conclusion oxadiazolylbiphenylpyridine is a promising scaffold that may be useful for the development of new antimicrobials to overcome antimicrobial resistant strains specially gram positive ones and fungi. 


\section{REFERENCES}

Clinical and Laboratory Standards Institute, C. (2007). Methods for antimicrobial susceptibility testing of anaerobic bacteria: CLSI Wayne, PA.

Clinical and Laboratory Standards Institute, C. (2008). Reference Method for Broth Dilution Antifungal Susceptibility Testing of Yeasts. Approved Standard - Third Edition, Wayne, PA, M27-A3

Clinical and Laboratory Standards Institute, C. (2012). Methods for dilution antimicrobial susceptibility tests for bacteria that grow aerobically. M7-A9. 9th ed.

Desai, N., Maheta, A., Rajpara, K., Joshi, V., Vaghani, H., \& Satodiya, H. (2014). Green synthesis of novel quinoline based imidazole derivatives and evaluation of their antimicrobial activity. Journal of Saudi Chemical Society, 18(6), 963971.

French, G. (2006). Bactericidal agents in the treatment of MRSA infections-the potential role of daptomycin. Journal of Antimicrobial Chemotherapy, 58(6), 1107-1117.

Geers, T. A., \& Donabedian, A. M. (1989). Comparison of broth microdilution and agar dilution for susceptibility testing of Neisseria gonorrhoeae. Antimicrobial Agents and Chemotherapy, 33(2), 233-234.

Mohammad, H., Mayhoub, A. S., Cushman, M., \& Seleem, M. N. (2015). Antibiofilm activity and synergism of novel thiazole compounds with glycopeptide antibiotics against multidrug-resistant staphylococci. J Antibiot (Tokyo), 68(4), 259-266. doi: 10.1038/ja.2014.142

Mohammad, H., Mayhoub, A. S., Ghafoor, A., Soofi, M., Alajlouni, R. A., Cushman, M., \& Seleem, M. N. (2014a). Discovery and characterization of potent thiazoles versus methicillin-and vancomycin-resistant Staphylococcus aureus. Journal of medicinal chemistry, 57(4), 1609-1615.

Mohammad, H., Mayhoub, A. S., Ghafoor, A., Soofi, M., Alajlouni, R. A., Cushman, M., \& Seleem, M. N. (2014b). Discovery and characterization of potent thiazoles versus methicillin-and vancomycin-resistant Staphylococcus aureus. J. Med. Chem., 57(4), 1609-1615.

Pankey, G., \& Sabath, L. (2004). Clinical relevance of bacteriostatic versus bactericidal mechanisms of action in the treatment of Gram-positive bacterial infections. Clinical infectious diseases, 38(6), 864-870.

Parmar, V. S., Sharma, N. K., Husain, M., Watterson, A. C., Kumar, J., Samuelson, L. A. Malhotra, S. (2003). Synthesis, characterization and in vitro 
anti-invasive activity screening of polyphenolic and heterocyclic compounds. Bioorganic \& medicinal chemistry, 11(6), 913-929.

Prakash, O., Hussain, K., Kumar, R., Wadhwa, D., Sharma, C., \& Aneja, K. R. (2011). Synthesis and antimicrobial evaluation of new 1, 4-dihydro-4pyrazolylpyridines and 4-pyrazolylpyridines. Organic and medicinal chemistry letters, $1(1), 5$.

\section{تصميم وتثييي مركبات جليدة غير متجانسة الحلقة للتظلب على المقاومة الميكروبية}

أحمد مصطفى عبد الثافى هلال ، عبد الرحمن صلاح ميهوب ، حماده السيد أبو الخير ، أشرف حسن بيومى

$$
\text { قسم الكيمياء العضوية ـ كلية الصيدلة (بنين) - جامعة الأزهر - القاهرة- مصر }
$$

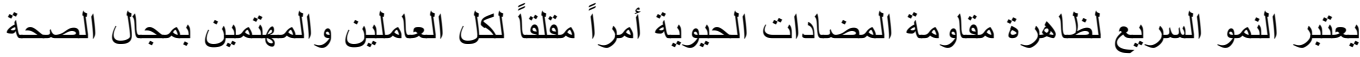

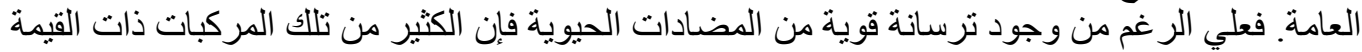

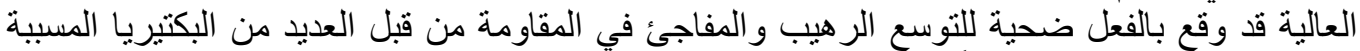

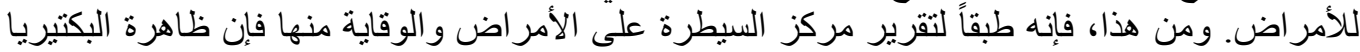

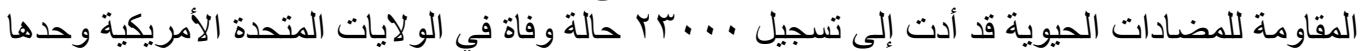

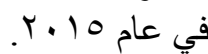
هذا وقد ثبت النشاط الواعد الذي يضطلع به مركبات الفينل ثيازول ضد مسببات الأمراض البكتئيرية

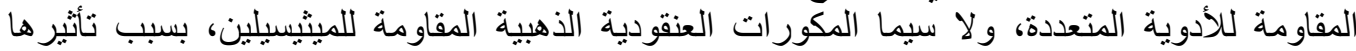

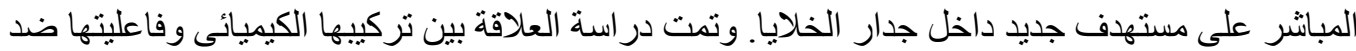

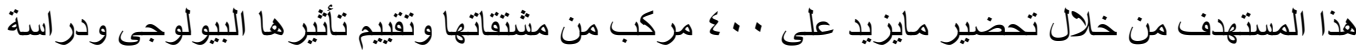
الحركية الدو ائية لبعضها.

هنا، قمنا بتصميم وتخليق ودراسة الناثير البيولوجى لسلسلة من مركبات الفينيل بيريدين التى ينتشابه

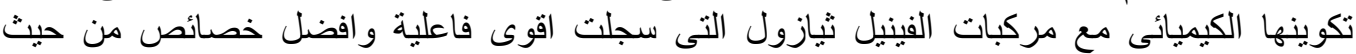

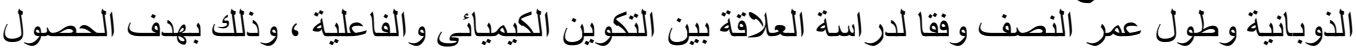

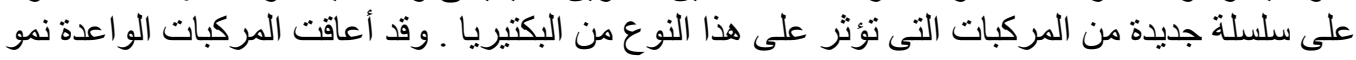

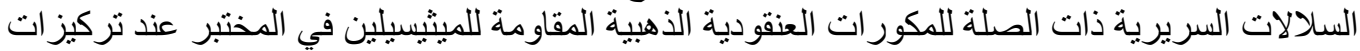

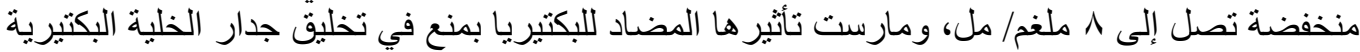

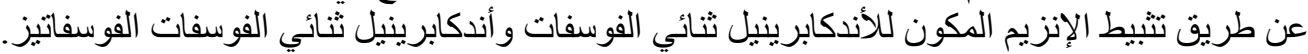

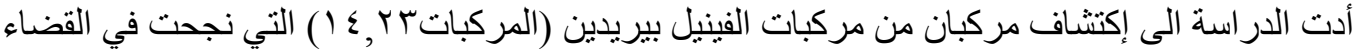

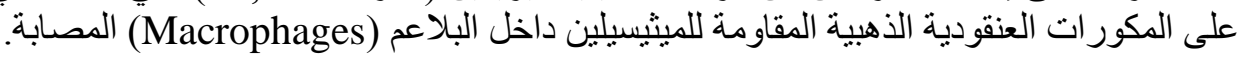

